\title{
PHOTOGRAMMETRY-BASED AUTOMATED MEASUREMENTS FOR TOOTH SHAPE AND OCCLUSION ANALYSIS
}

\author{
V. A. Knyaz ${ }^{\text {a }}$ A.V. Gaboutchian ${ }^{\text {b*, }}$ \\ ${ }^{a}$ State Research Institute of Aviation System (GosNIIAS), 125319 Moscow, Russia - knyaz@gosniias.ru \\ ${ }^{\mathrm{b}}$ Moscow State Medical-Stomatological University, Moscow, Russia - armengaboutchian@mail.ru
}

Commission V, WG V/5

KEY WORDS: Photogrammetry, 3D reconstruction, Automation, Segmentation, Dentistry, Odontometry, Occlusiometry, Odontotomy.

\begin{abstract}
:
Tooth measurements (odontometry) are performed for various scientific and practical applications, including dentistry. Present-day techniques are being increasingly based on 3D model use that provides wider prospects in comparison to measurements on real objects: teeth or their plaster copies. The main advantages emerge through application of new measurement methods which provide the needed degree of non-invasiveness, precision, convenience and details. Tooth measurements have been always regarded as a time-consuming research, even more so with use of new methods due to their wider opportunities. This is where automation becomes essential for further development and implication of measurement techniques. In our research automation in obtaining 3D models and automation of measurements provided essential data that was analysed to suggest recommendations for tooth preparation - one of the most responsible clinical procedures in prosthetic dentistry - within a comparatively short period of time. The original photogrammetric 3D reconstruction system allows to generate 3D models of dental arches, reproduce their closure, or occlusion, and to perform a set of standard measurement in automated mode.
\end{abstract}

\section{INTRODUCTION}

Measurements of teeth are more often becoming a key point for successful dentistry treatment. However existing measurement methods do not always provide sufficient accuracy and convenience for dentists. Intra-oral measurements are problematic, so traditional means require the use of dental arches' plaster copies. But even so the required degree of objectivity, versatility and accuracy of studies remain unattainable. In some cases it is necessary to carry out large number of measurements and to analyze the results; nevertheless traditional methods do not offer any automation.

An adequate analysis of tooth shape and closure needs a set of measurements and estimation of tooth position in relation to neighboring and opposing teeth. Performing such measurements using plaster casts of dental arches is challenging. Thereby a photogrammetric measuring system was developed for providing a convenient and efficacious measurement process. It works with accurate 3D models of dental arches produced by original photogrammetric $3 \mathrm{D}$ reconstruction system and allows to register teeth occlusions and to perform a set of standard measurement in automated mode.

Measurements have become indispensable in most scientific and practical activities. Different objects, including teeth, are subjected to measurements. One part of measurement techniques, known as photogrammetry, provides obtainment of 3D images of teeth: intra- or extra-oral (Knyaz et al, 2007; Mitchell and Chadwick, 2008). Another part of methods odontometric - provides determination of sizes, dimensions and proportions of teeth, their copies or images (Zubov, 1968).
Positive results of combining these two different approaches to measurements are presented below.

Odontometry include various methods and applications in dentistry. It is put into practice in tooth preparation in prosthodontics (Marxkors, 2003), studies of teeth durability (Khera et al, 1990), studies of attrition (Khan and Young, 2011), in tooth modelling (Lomiashvili and Ayupova, 2008) and etc. Odontometric methods are widely used in anthropology. They become a part of ethnological, archaeological, genetic, gender, racial and other studies (Zubov, 1973; Townsend et al, 2012; Pilloud et al, 2014).

Our research refers to preparation of occlusal surface of premolars and molars. This is a part of clinical procedure of reshaping teeth for subsequent crowning. Due to specific functions of occlusal surfaces, the shape of a prepared tooth, as well as its' correct interrelation with opposing teeth, influence treatment results. Preparation techniques accepted in dentistry recommend that a tooth preparation procedure should provide conditions for smooth crown placement and, at the same time, similarity to an intact tooth. Obviously, dentists are not aimed to duplicate occlusal surface minute details on prepared teeth. In most cases there are no chances to detect them due to severe damage of teeth subjected to prosthetic treatment. Nevertheless, the most expressed features of tooth structure must be cut on prepared teeth to avoid erroneous flattening of occlusal surface. To estimate that features we measured teeth of different groups: upper and lower, molars and premolars. Traditional measurements techniques aimed on estimation of extreme tooth dimensions could not provide necessary data. Hereby a specific odontometric method was elaborated.

\footnotetext{
* Corresponding author.
} 
In terms of our research subject proportions of tooth structures were measured and average values, specific for studied groups of teeth, were calculated. For other parts of the research distribution of marked features along the coronal part of teeth was estimated. Therefore we applied a method of measurements, based on sectioning the whole occlusal surface extension by series of parallel planes (the suggested name for the method - odontotomy). Photogrammetrically obtained 3D models of dental arches were used for sectioning (Knyaz et al, 2007). Uniformity of the obtained tooth contours allowed the use of identical measurement landmarks for all sections (Gaboutchian, 2011). Described approaches helped to increase the degree of objectivity and details of odontometry in our research and to suggest a new method of measuring opposing teeth closure - occlusiometry. One of the most notable disadvantages of the odontotomic method is in increase of quantity of measurements related to any tooth and subsequent increase of time consumption for such a research (even though the most of odontometric studies are well known as time consuming). Thus the necessity of measurement automation arose. The initial step of automation refers to measurement landmark estimation on $2 \mathrm{D}$ tooth contours. This facilitates to speed-up the study and to increase measurement precision, as results become predominantly dependent on accuracy of photogrammetry but not operator skills. Further automation regards landmark estimation on 3D images of teeth.

\section{AUTOMATED GENERATION AND PROCESSING OF DENTAL ARCH 3D MODELS}

Different techniques are used for tooth measurements including manual measurements of real teeth, manual measurements of tooth plaster copy (Figure 1), measurements performing with special software (Paredes et al, 2015; Naidu and Freer, 2013 a, b) working with $2 \mathrm{D}$ images or $3 \mathrm{D}$ digital models of teeth (Figure 2). They are found to be suitable for carrying out measurements, being compared to measurements by means of conventional hand instrument (Paredes et al, 2015, Zilberman et al, 2003) but still require a lot of manual operation to obtain the needed measurement results.

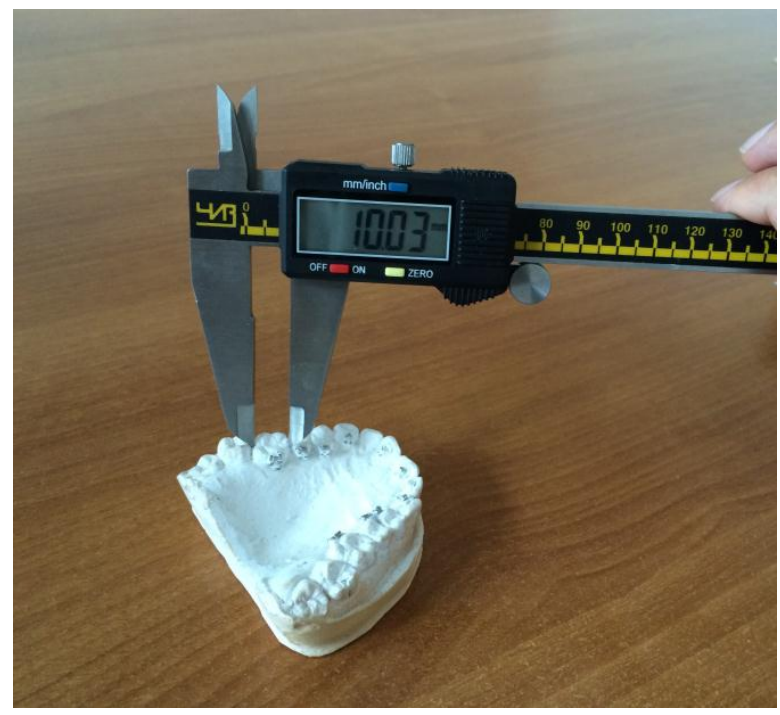

Figure 1. Measurements on plaster cast dental arch

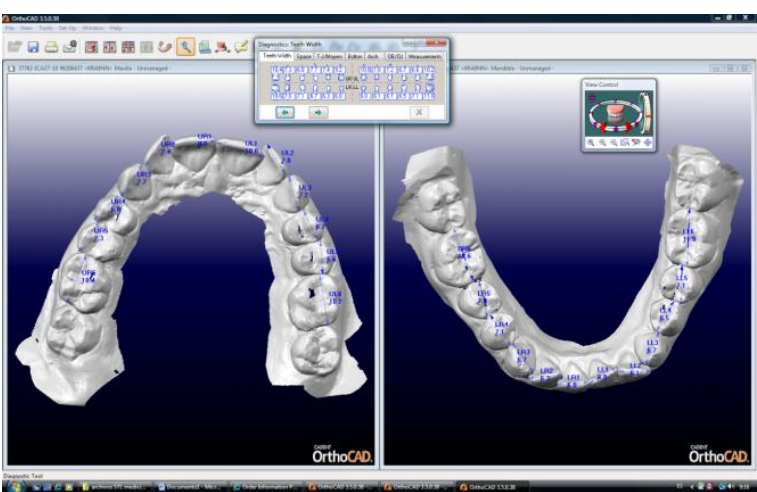

Figure 2. Measurements on 3D models of dental arches

Photogrammetric methods allow development of a complex technique for automated tooth measurements, starting from accurate 3D digital model generation and finalizing by automated extraction of the required tooth parameter.

The main steps of automated measurement process are the following: accurate 3D reconstruction of teeth arch, 3D model segmentation into a set of separate teeth, individual tooth dimensions determination, indication of anthropological reference points based on tooth structure features, definition of a coordinate system for separate teeth, measurements of defined parameters such as mesial-distal and vestibule-oral dimensions, differences in height of reference anthropological points, distances between antagonists' reference points, etc.

\subsection{Teeth occlusion registration}

For automated dental arch 3D model generation original photogrammetric system was used (Knyaz, 2012). It includes two digital high resolution cameras, high resolution structured light projector and PC-controlled rotation positioning stage. It allows producing high resolution 3D models of dental arches in automated mode (Figure 3).

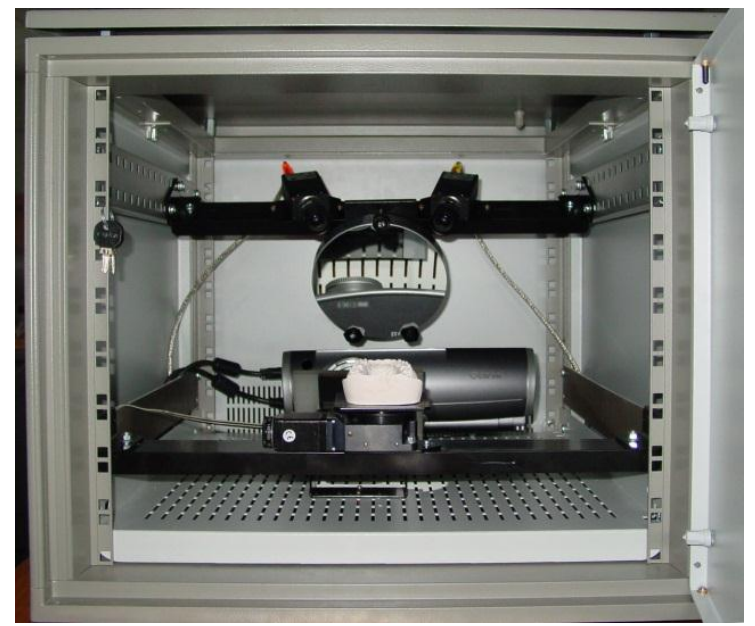

Figure 3. Photogrammetric system for 3D reconstruction of dental arches

The configuration of photogrammetric system includes two digital IEEE 1394 cameras of high resolution (2 Mpixel), structured light projector with resolution of 1440x1080 pixels and a PC-controlled rotation positioning stage. For automated 
corresponding problem solution coded light was applied to provide robust and fast scanning.

The system calibration was performed by means of original technique for camera interior and exterior orientation parameters estimation and for determination parameters of the positioning stage rotation axis (Knyaz and Zheltov, 2005). Special calibration test field with coded targets was used for determination of the system parameters in automated mode. The calibration technique provided residuals of co-linearity conditions for the reference points after least mean square estimation at the level of $0.005 \mathrm{~mm}$. This accuracy is quite adequate for the concerning problem.

For dental arch 3D model generation a set of plaster model scans was acquired in various positions. The number of scans and their orientation are the subject of user choice. All scans are transformed to coordinate system using the results of rotation axis parameters estimation. This first order scans alignment serves as an initial approximation for accurate scan registration by iterative closest point algorithm (Besl and McKay, 1992). After scan merging a single mesh 3D model is generated using interpolating mesh algorithm (Curless and Levoy, 1996).

The described automated procedure requires about 5 minutes for dental arch 3D model generation. The resulting 3D model provided accuracy of $0.03 \mathrm{~mm}$ and includes about 1.000 .000 points. Both, upper and lower, dental arches' 3D models were used for subsequent tooth shape and occlusion analysis.

\subsection{Teeth occlusion registration}

For occlusion registration special mode is used. In occlusion registration mode 3D models of upper and lower dental arches are placed in working space of scanning system. Then a 3D scan of stone cast dental arches in previously registered occlusal position is captured. This 3D scan is used for placing 3D models of upper and lower teeth arches in corresponding position (Figure 4).

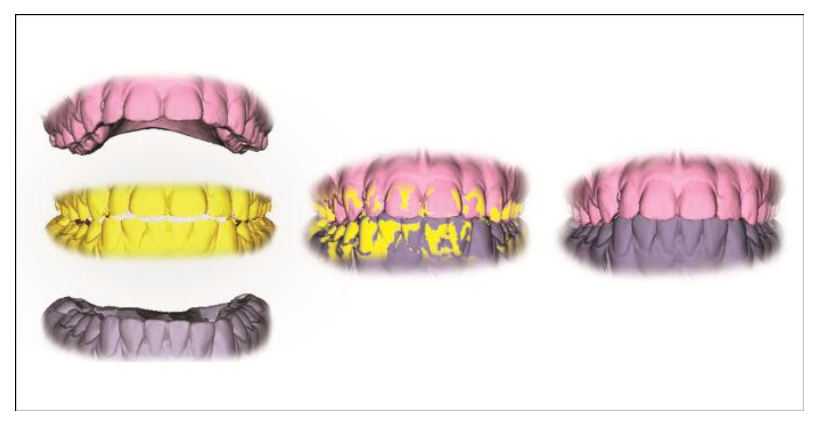

Figure 4. Stages of scanning and positioning for 3D models of opposing dental arches

To reproduce the position of dental arches by means of 3D models we used an articulator - a device applied in dentistry for simulating dental arch position and closure (occlusion) for diagnostics and prosthesis construction. Plaster models were installed in the articulator in centric occlusion position and scanned. Silicon moulds were made for right and left occlusions' reproduction. Thus 3D models of dental arches were provided for measurements and analysis of tooth structure and occlusion.
For providing further phases of the research, tooth preparation on plaster casts was carried out. Prepared teeth were scanned and their 3D images were combined with images of appropriate dental arches by means of the developed technique. Subsequently, prepared plaster teeth were used for wax modelling of tooth crowns. These crowns were scanned and combined with initial images respectively. As a result we obtained multipart 3D models of opposing dental arches in three occlusal positions combined with images of prepared teeth and crowns. All parts of these complex models were positioned in single coordinate system.

Therefore dentists had at their disposal 3D models of dental arches installed in different positions for performing all necessary studies of opposing occlusal surfaces and occlusion. In order to achieve correct spatial placement of sections, each pair of opposite dental arches was oriented with respect to a plane in a manner similar to their position in an adjustable articulator.

\subsection{D model segmentation}

For dental arch 3D model segmentation an algorithm based on gradients analysis of dental arch in 2D and 3D modes was applied (Figure 5). Automated detection of anthropological reference points is performed using the results of the gradients analysis for feature detection and model-based approach. Then the detected reference points are used for defining tooth system of coordinates. After that a set of predefined teeth parameters is determined in automated mode.

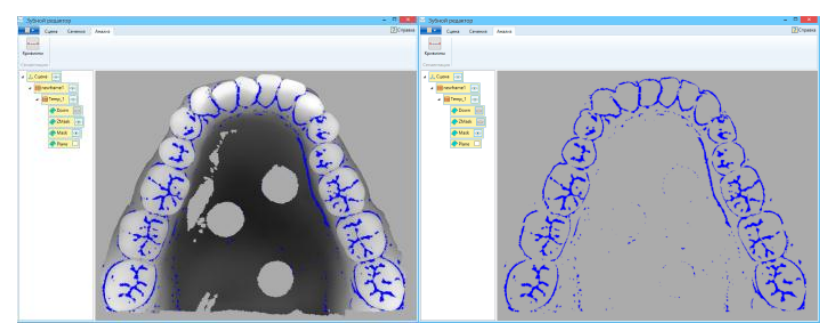

Figure 5. Results of dental arch 3D model segmentation

\subsection{Automated measurements of tooth parameters}

After segmentation, 3D models of dental arches were divided into a set of separate tooth models. For every tooth a set of predefined parameters such as mesio-distal and vestibular-oral dimensions, positions of anthropological points and distances between them can be calculated in automated mode.

\section{ODONTOMETRIC TECHNIQUES}

The majority of the existing odontometric methods are predominantly directed to estimation of maximal tooth sizes in different dimensions. Such measurements provide important information but do not reveal the variety of tooth shapes and relief. For this reason, in line with methods of measurements, methods of description (known as odontoglyphics) are applied in odontology (Zubov, 1977). Another common trait of odontometric methods: traditional or alternative (Hillson et al, 2005), applied to real objects or 3D models, is their subjectivity based on expert estimation of measurement landmarks on tooth surface. Studies show intra- and inter-observer errors (Paredes et al, 2015). However existing discrepancies are accepted as satisfying, as an example, for clinical application in 
orthodontics (Berman, 2010; Hortona et al, 2010; Paredes et al, 2015; Reuschi et al, 2015, Zilberman et al, 2003). But at the same time we are still facing the deficiency of more objective means for setting measurement landmarks on tooth surfaces; no matter we use traditional real object or modern 3D images for measurements. Nevertheless in terms of prospects computeraided techniques have a serious advantage in comparison to traditional. They allow creating algorithms for automated landmark setting that can increase measurement objectivity and equate measurement precision to the precision of the $3 \mathrm{D}$ image.

Precision remains a feature of high demand of any measurement technique. Regarding prosthetic dentistry, progress in treatment quality has been always associated with introduction of new, precision increasing technologies in clinical and laboratory methods of prosthesis manufacturing. In our days this shows by development of clinical and laboratory CAD/CAM technologies that are undoubtedly based on measurement techniques (CEREC, Zirkonzanh, etc). Such techniques usually suggest a limited choice of tooth shapes and their automated positioning in accordance to available space; option of "manual" reshaping by dentist or dental technician is also available. At the same time, taking into consideration the difference of measurement techniques mentioned in this article introduction, we cannot find evidence of regular use of tooth measurements and of their results' analysis during prosthesis manufacturing for making treatment more precise and predictable.

\subsection{Odontomy}

On the basis of tooth preparation research we would like to demonstrate positive effect of measurement techniques' improvement after a short description of the initially applied method (Batrak et al, 2010). Odontotomic method was used to obtain tooth contours for subsequent measurements: 20 parallel sections between two points on mesial and distal marginal ridges were constructed on every examined tooth (Figure 6). We would like to mention that the choice of points (landmark) was expert-based at this stage, i.e. subjective; sectioning automated. Even distribution of the collected data through the occlusal surface extension provides improvement of the study objectivity. Directions of the sections (vestibular-oral) provided depiction of tooth structures that were subjected to study in the research.

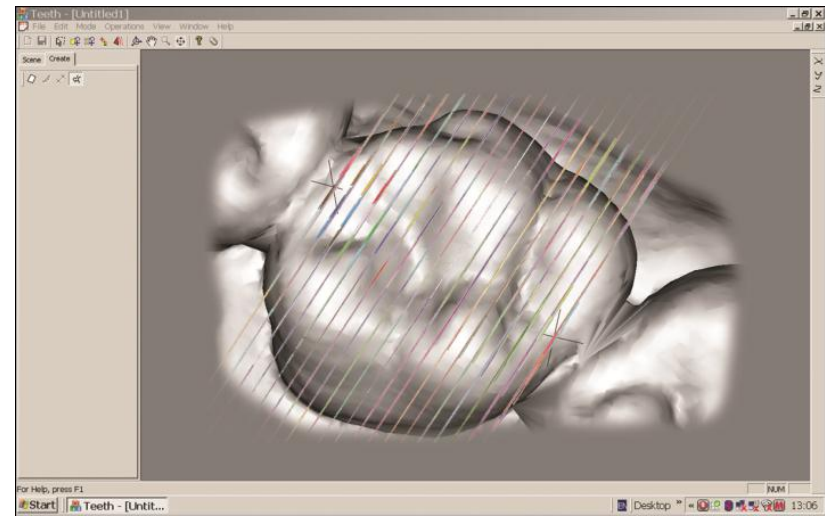

Figure 6. Odontotomy in vestibular-oral direction

\subsection{Odontometry}

Tooth contours obtained by sectioning were transformed to $2 \mathrm{D}$ images for geometric constructions and measurements based on similar landmarks on all sections. We name them unconditional landmarks on premolar and molar contours (Figure 7).

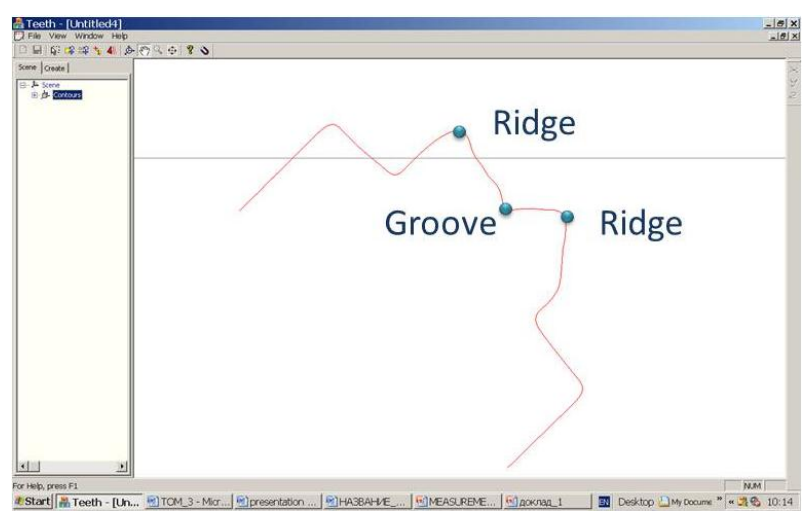

Figure 7. Measurement landmarks on a tooth contour

Odontometric part of the research included estimation of the following parameters: vestibular-oral dimensions of teeth, cusps and cusp slopes and mesio-distal groove depth (Figure 8). Landmark positioning was performed in "manual" mode, but we would like to emphasize the convenience of digital measurement techniques which provide operator with different useful functions, such as: image magnification, re-measuring option, automated data saving, etc. In some parts of odontometric research we accepted as a measurement result the average of three consequent measurements to minimize research subjectivity. However it seems evident that setting of measurement landmark position on a flat image can be performed with higher precision than on a volumetric object. This can be achieved by combination of odontotomic and odontometric methods that we used in the research. The collected odontometric data served for calculating the proportions of tooth structures important in terms of preparation.

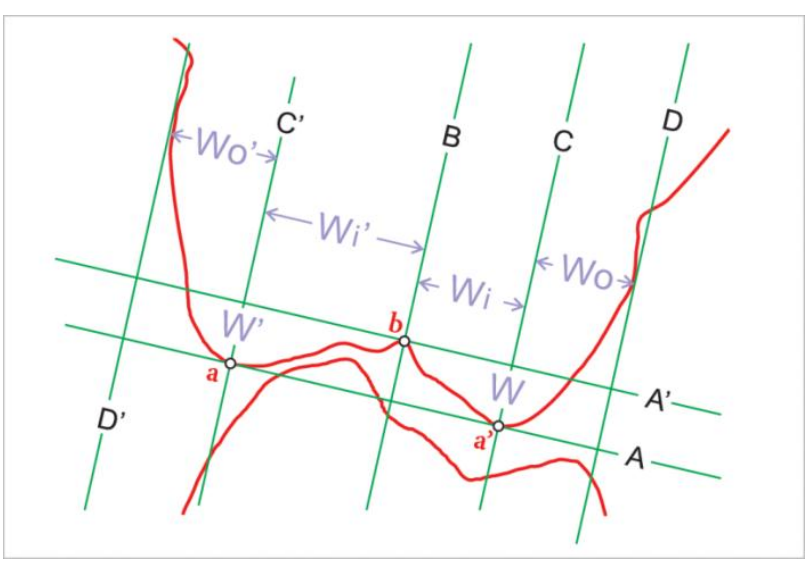

Figure 8. Odontometry on a tooth contour in vestibular-oral dimension

\subsection{Occlusiometry}

Landmarks, similar to those used for odontometry, allowed to measure tooth closure as well. Utilizing odontotomy of dental arched closed in maximal intercuspation, contours of opposing teeth were obtained for further study. These measurements were included in the occlusiometric part of our research. Within its confines cusp penetration and deviation in relation to opposite 
anatomic occlusal surface were measured (Figure 9 a, b). The collected occlusiometric data served for calculation of parameters that define interrelations of opposite teeth structures important in terms of correct preparation.

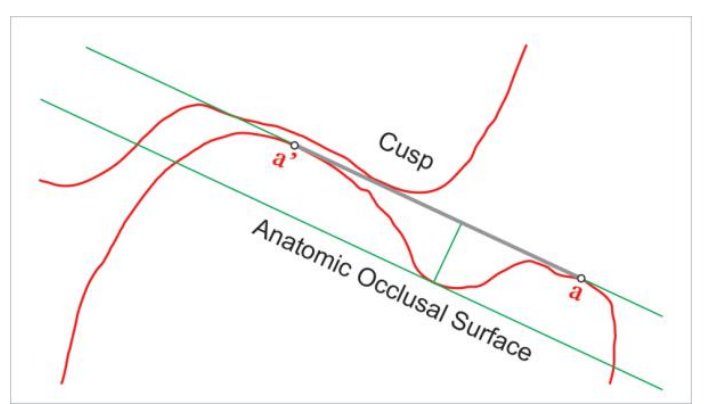

Figure 9a. Occlusiometry on tooth contours. Example of cusp penetration measurements.

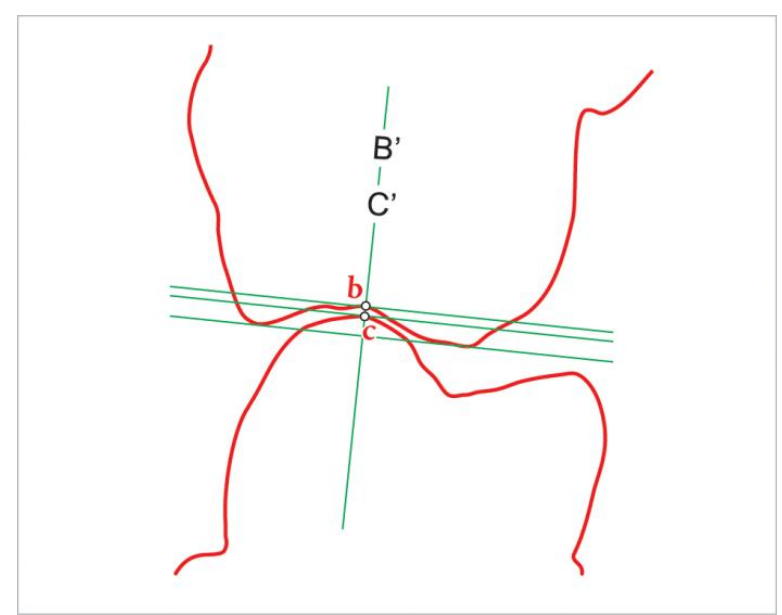

Figure 9 b. Occlusiometry on tooth contours. Example of cusp deviation measurements.

\subsection{Odontoscopy}

Another study involved combined contours of teeth, prepared teeth and crowns (Figure 10). Although this method of study was not initially applied as an odontometric method, we represent it in this chapter as subsequently subjected to automation.

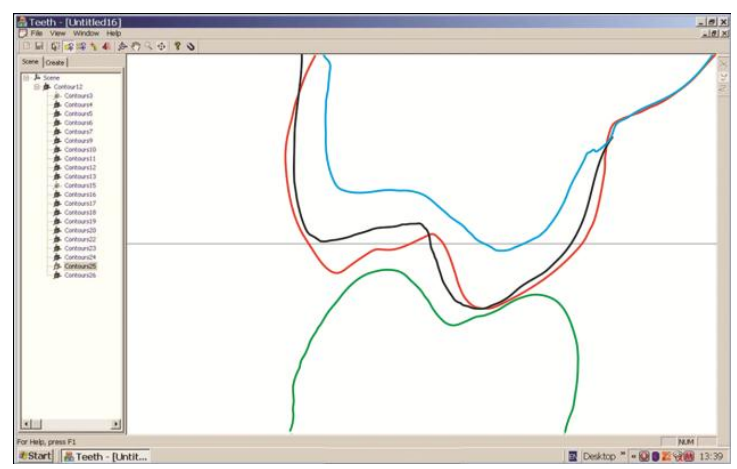

Figure 10. Combined contours of teeth, prepared teeth and crowns
Experimental tooth preparation was performed on plaster models in accordance with existing recommendations; crown contours were modelled in wax. Combined images initially were sectioned and studied only visually - not alike single or opposite teeth contours. This, odontoscopic, stage of research revealed the necessity of preparation of specific areas on molars for prevention of crown thinning or over-contouring.

The abovementioned methods were applied to the 3D images' study within the research confines. Initially only sectioning was performed automatically. Our further improvement of measurement methods refers to automation of "manual" measurements and visual studies.

The main stages of measurement process were following: dental arch 3D model segmentation into a set of separate teeth, individual tooth dimensions determination, indication of anthropological reference points based on tooth structure, definition of coordinate system for every separate tooth, measurements in mesial-distal and vestibule-oral dimensions, determination of distances and differences between reference landmarks.

\section{AUTOMATION OF ODONTOMETRY}

As mentioned before, after setting two initial landmarks on tooth surface, odontotomy had been carried out in automated mode. Sections, after their quantity setting, were constructed with even intervals. An update of this function includes the following features: increase of number of possible sections from 30 to 50 - between landmarks and their interval setting option. The first feature enables a more detailed study. The second gives opportunity to equalize conditions of inter-teeth studies and comparisons.

Another direction in measurement automation concerns landmark setting on 2D contours (Figure 11). Algorithms of automated point detection were applied to single tooth contours. Point detection simultaneously provides data on measurable parameters straight away. The parameters are: vestibular-oral dimension of inner slopes and mesio-distal groove depth.

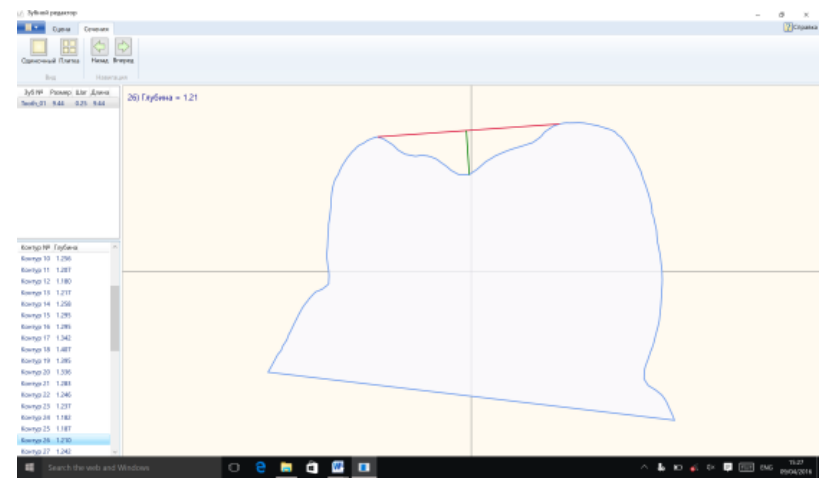

Figure 11. Automated landmark setting and measurements on tooth contours

Automation regards combined contours' analysis as well. To estimate areas of insufficient preparation, instead of visual study, automated detection was applied. Pairs of contours were analysed: intact tooth - prepared tooth; intact tooth - crown; prepared tooth - crown (Figure $12 \mathrm{a}, \mathrm{b}, \mathrm{c}$ ). 


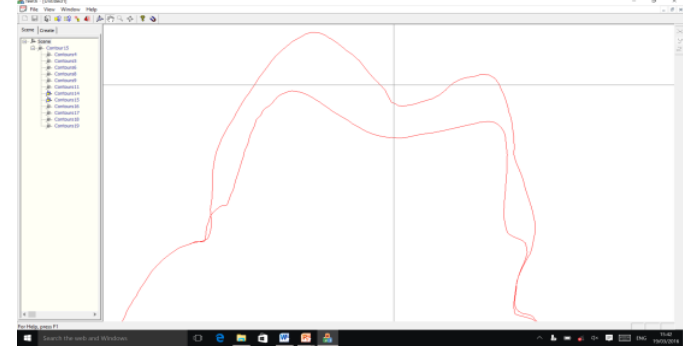

a

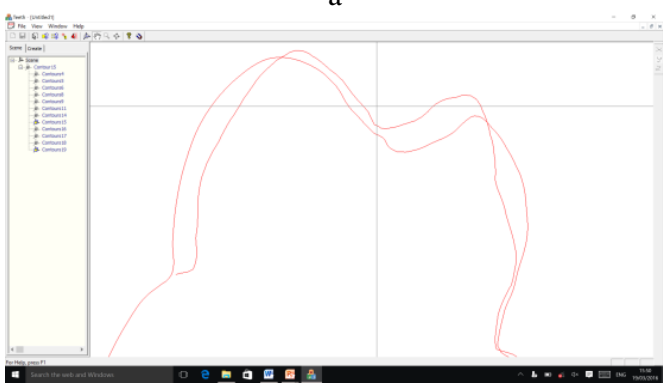

$\mathrm{b}$

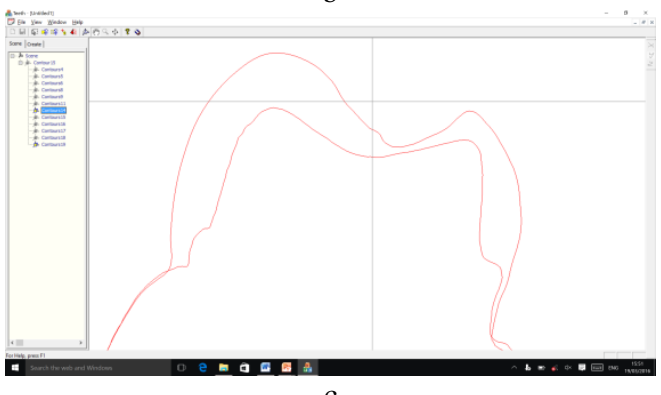

Figure 12. Combined contours for automated feature detection: $\mathrm{a}$ - intact tooth/prepared tooth; $\mathrm{b}$ - intact tooth/crown; c prepared tooth/crown

Measurement automation required application of gradients analysis algorithms on different stages of measurement process. For dental arch 3D model segmentation an algorithm based on gradients analysis of dental arches in 2D and 3D modes was applied. Automated detection of anthropological measurement reference landmarks was performed using the results of the gradients analysis for feature detection and model-based approach. Then the detected reference points were used for defining tooth coordinates' system. After that a set of predefined tooth parameters was determined in an automated mode.

\section{AUTOMATION RESULTS}

Our research initially included analysis of 4260 sections of teeth: 2640 - for odontometry; 1380 - for occlusiometry; 240 for odontoscopy. Tooth and occlusion measurement research included over 13.5 thousand geometric constructions. Measurement results were subjected to analysis and over 15 thousand indicators characterizing teeth and occlusion. They served for grounding of preparation recommendations in 5 groups of teeth: upper premolars, lower first and second premolars, upper molars, and lower molars. Each of them was divided to two subgroups of teeth with or without distinct attrition. Attrition, in its turn, was estimated not only visually but through measurements as well (occlusal surface relief indicator was determined between 3.09 and 12.062). An example of tooth shape suggested for preparation with its proportions is shown on Figure 13.

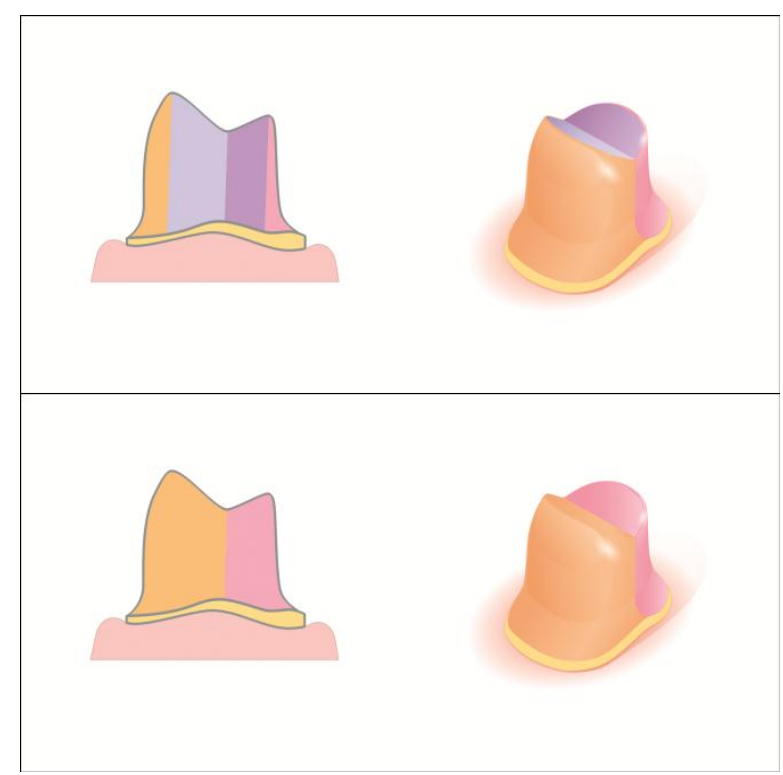

Figure 13. Lower second premolar preparation contour and shape view

As we estimated during our initial research the first measurement, including 20 section odontotomy, required about 45 seconds; each of the following geometric construction about 30 seconds for obtaining one indicator. Automated measurement software that we have at our disposal today allows 50 section odontotomy and 50 measurements' results in 10 seconds. Some studies have shown that measurements on 3D models can be done faster than on real objects (Berman, 2010; Reuschi et al, 2015) but measurement automation shows significantly higher speed-up results.

For automated analysis of teeth, prepared teeth and crowns seven combined 3D models of molars were chosen (Table 1). This study allowed detection of insufficient preparation areas, crown over-contouring and other features that previously were subjects of visual analysis only. Results of visual study were confirmed by automated contour analysis. Automation of contour needs less time and allows simultaneous multi-feature analysis.

\begin{tabular}{|l|c|c|}
\hline Molars & Upper & Lower \\
\hline Right & 3 & 0 \\
\hline Left & 1 & 3 \\
\hline
\end{tabular}

Table 1. Combined 3D models of molars

\section{CONCLUSION}

At the present time photogrammetry has become widely used in many dental applications for precise reproduction of teeth and dental arch shapes and closure.

Combination of tooth measurements with photogrammetric methods showed its ability to provide essential data for a specific research in dentistry. At the same time the research 
revealed a high potential for use of measurements in clinical and laboratory procedures in prosthetic dentistry and other purposes. Measurement automation shows its ability to speedup studies which is important for making odontometry applicable in everyday practice. We can assume that automation can increase objectivity of measurements. This is important for different applications where odontometry is already in use. Besides, we can forecast involvement of automated odontometry in other research directions due to increase of its accessibility and reliability. Our approaches to odontometry can be applied to measurement of other objects with irregular relief - not teeth.

Our proximate plans include widening the variety of measurable parameters and further landmark detection automation on $2 \mathrm{D}$ and $3 \mathrm{D}$ images.

\section{ACKNOWLEDGEMENTS}

The work was performed with the support by Grant 15-0899580a of Russian Foundation for Basic Research (RFBR).

\section{REFERENCES}

Batrak, I.K., Bolshakov, G.V., Gaboutchian, A.V., 2010. Method of measurement of teeth, tooth cusps and their inner and outer inclines. Patent for invention № 2438617.

Berman, G., 2010. Tooth size measurements and Bolton analysis for fast-set plaster models versus computer-based models rendered from dual pour alginate impressions. MDS thesis. Pittsburgh.

Besl, P.J., McKay, N.D., 1992. A method for registration of 3D shapes, IEEE Transactions on Pattern Analysis and Machine Intelligence, vol. 14, no.2, pp. 239-256

Curless, B., Levoy, M., 1996. A Volumetric Method for Building Complex Models from Range Images. SIGGRAPH 96 Conference proceedings, pp. 303-312

Gaboutchian A.V., 2011. Clinical-experimental study of tooth occlusal surface preparation for prosthetic treatment by fixed prosthesis. PhD thesis synopsis, Moscow, MSMSU.

Hillson, S., FitzGerald, C., Flinn, H., 2005. Alternative Dental Measurements: Proposals and Relationships With Other Measurements. Am J Phys Anthropol 126:413-426.

Hortona, H.M.I., Millerb J.R., Gaillardc P.R., Larsond B.E., 2010. Technique Comparison for Efficient Orthodontic Tooth Measurements Using Digital Models. Angle Orthodontist, Vol 80, No 2254-261

Khan, F., Young W.G., 2011. Tooth wear: The ABC of the worn dentition. Wiley-Blackwell.

Khera, S.C., Carpenter, C.W., Staley, R.N., 1990. Anatomy of cusps of posterior teeth and their fracture potential. -JPD, v 64, № 2, p. 139-147.

Knyaz, V., 2012. Image-Based 3D Reconstruction and Analysis for Orthodontia. Int. Arch. Photogramm. Remote Sens. Spatial Inf. Sci. Vol. XXXIX-B3, pp. 585-589
Knyaz, V.A., Zheltov, S.Yu., 2005. On-line calibration technique for mobile robot. EOS Conference on Industrial Imaging and Machine Vision, Munich, Germany, June 13-15, pp. 95-97

Knyaz, V., Zheltov, S., Gabuchyan, A., Bolshakov, G., 2007. Photogrammetric system for automated teeth arches 3D models generation and teeth occlusion analysis. Optical $3 D$ Measurement Techniques VIII, Zurich, Vol. I, pp. 299-304.

Lomiashvili, L.M., Ayupova, L.G., 2008. The art of modelling and restoration of teeth. Med. Kniga.

Pilloud, M.A., Hefner, J.T., Hanihara, T., Hayashi, A., 2014. The Use of Tooth Crown Measurements in the Assessment of Ancestry. J Forensic Sci, November, Vol. 59, No. 6.

Marxkors, D., Marxkors, R., 2003. Tooth preperation for fixing crowns. Novoe v stomatologii, № 1, pp. 73-76.

Mitchell, H.L., Chadwick, R.G., 2008. Challenges of photogrammetric intra-oral tooth measurement. The International Archives of the Photogrammetry, Remote Sensing and Spatial Information Sciences. Vol. XXXVII. Part B5. Beijing.

Naidu, D., Freer, T.J. 2013a. The evidence supporting methods of tooth width measurement: Part I.Vernier calipers to stereophotogrammetry. Aust Orthod J. Nov; 29(2):159-63.

Naidu, D., Freer, T.J. 2013b. The evidence supporting methods of tooth width measurement: Part II. Vernier calipers to stereophotogrammetry. Aust Orthod J. 2013 Nov; 29(2):164-9.

Paredes, V., Tarazona, B., Zamora, N., Hernandez, Y., San José, V., 2015. A Comparative Study of Reproducibility, Reliability and Accuracy in Measuring Mesiodistal Tooth Size Using Four Different Methods - 2D Digital, 3D CBCT, 3D Segmented and 3D Intraoral Scanner Method. Issues in Contemporary Orthodontics. http://dx.doi.org/10.5772/59366.

Reuschi, R.P., Heuer, W., Stiesch, M., Wenzel, D., Dittmer, M.P., 2015. Reliability and validity of measurements on digital study models and plaster models. Eur J Orthodont. doi: http//dx.doi.org/10.1093/ejo/cvj001

Townsend, G., Kanazawa, E., Tokuyama, H., 2012. New Directions in Dental Anthropology: paradigms, methodologies and outcomes. University of Adelaide Press.

Zilberman, O., Huggare, J., Parikakis, K., 2003. Evaluation of the Validity of Tooth Size and Arch Width Measurements Using Conventional and Three-dimensional Virtual Orthodontic Models. Angle Orthod;73:301-306.

Zubov, A.A., 1968. Odontolody. Anthropological study technique. Nauka.

Zubov A.A., 1973. Ethnical odontology. Nauka.

Zubov, A.A., 1977. Odontoglyphics: the laws of variation of the human molar crown microrelief. Orofacial grows and development. Hague. 